\title{
Mobile health-monitoring system through visible light communication
}

\author{
Yee-Yong Tan and Wan-Young Chung* \\ Department of Electronic engineering, Pukyong National University, Busan 608-737, Korea
}

\begin{abstract}
Promising development in the light emitting diode (LED) technology has spurred the interest to adapt LED for both illumination and data transmission. This has fostered the growth of interest in visible light communication (VLC), with on-going research to utilize VLC in various applications. This paper presents a mobile-health monitoring system, where healthcare information such as biomedical signals and patient information are transmitted via the LED lighting. A small and portable receiver module is designed and developed to be attached to the mobile device, providing a seamless monitoring environment. Three different healthcare information including ECG, PPG signals and HL7 text information is transmitted simultaneously, using a single channel VLC. This allows for a more precise and accurate monitoring and diagnosis. The data packet size is carefully designed, to transmit information in a minimal packet error rate. A comprehensive monitoring application is designed and developed through the use of a tablet computer in our study. Monitoring and evaluation such as heart rate and arterial blood pressure measurement can be performed concurrently. Real-time monitoring is demonstrated through experiment, where non-hazardous transmission method can be implemented alongside a portable device for better and safer healthcare service.
\end{abstract}

Keywords: Visible light communication, light emitting diode, mobile-health monitoring system, healthcare

\section{Introduction}

In recent years, the research to discover alternative energy-efficient products has been rigorously on-going, especially in the lighting area. The purpose is to halt the deceleration of energy consumption, which will lead to high-usage of the fossil fuels and thus damaging the environment. Lighting, being the dominant sector for energy consumption, has garnered interest to develop energy efficient light source. This leads to the development of the high-efficacy light emitting diode (LED). The importance of the LED technology is highlighted by the Ministry of Trade, Industry and Energy in South Korea, where companies are prohibited from importing or producing incandescent light bulbs from January 2014 [1]. The evaluation conducted by the United States Department of Energy further corroborated the importance of LED technology, as shown in Table 1 [2]. There is superiority in terms of power consumption and operating lifetime in LED technology as compared to traditional lighting technology, such as incandescent and fluorescent lighting. This clearly shows the potential of the LED lighting technology to replace all the conventional illumination tools in various aspects of our lives.

\footnotetext{
${ }^{*}$ Corresponding author: Wan-Young Chung, Department of Electronic engineering, Pukyong National University, Busan 608-737, Korea. Tel.: +82-51-629-6223; Fax: +82-51-629-6210; E-mail: wychung@pknu.ac.kr.
}

0959-2989/14/\$27.50 @ 2014 - IOS Press and the authors. 
Table 1

Performance of the conventional and LED lighting technology

\begin{tabular}{llll}
\hline Lamp Type & Watts & Lumens & Operating Lifetime (hours) \\
\hline Incandescent & 60 & 900 & 1000 \\
\hline Compact florescent lamp & 15 & 900 & 8500 \\
\hline LED (2011) & 12.5 & 800 & 25000 \\
\hline LED - future (2015) & 5.8 & 800 & 40000 \\
\hline
\end{tabular}

Another major concern these days are regarding the compatibility of medical devices in healthcare centers with the incorporation of wireless technology [3]. Wireless technology like radio frequency (RF) technology has always been associated with the emission of electromagnetic interference (EMI) [4]. Intrusion of the EMI jeopardize the quality of medical monitoring, as the accuracy and efficiency of data transmission is crucial for the medical staff to provide corresponding measures or treatment, based on the real-time information received [5]. Furthermore, the prolong usage of RF wireless technology in the medical field has been in debate due to the emission of RF radiation that would deteriorate the health of the patient.

Visible light communication (VLC) is the usage of visually-perceived electromagnetic wave to transmit information. It is a form of optical wireless communication that utilizes the visible light spectrum from 380 to $780 \mathrm{~nm}$. The fast switching capability of the LED enables the transmission of data through VLC, by modulating the intensity of the optical sources in a very fast manner [6]. The unregulated bandwidth for VLC also proves to be an advantage, providing freedom in research and implementation without any restriction [7]. The advantages possessed by VLC make it a potential candidate as an alternative wireless technology. It maintains the similar characteristics as the conventional RF technology, while eliminating any disadvantages held by the RF technology, especially in the healthcare industry.

Researches implementing healthcare in mobile devices have intensified over the years [8]. Challa et al. developed a remote monitoring system where physician can remotely monitor the condition of the patients [9]. Gradl et al. developed a system to detect arrhythmia using electrocardiograph (ECG) signal via Android-based mobile system [10]. Alqassim et al. presented a method to use mobile device to detect symptoms of the sleeping disorder [11].

In this study, we aim to develop a mobile-health monitoring system using VLC as the data transmission method. This system comprises of the following aspects: (1) transmission of healthcare information via VLC; (2) real-time monitoring of the biomedical signals and patient information on the mobile device; (3) analysis of the biomedical signals received on a mobile device; (4) An alert function where a message can be sent instantaneously to a physician if there are any complications detected.

\section{System design and implementation}

In general, the mobile health-monitoring system using VLC transmission aims to eliminate the need for RF technology to be implemented in healthcare centers, such as a hospital. The transmission of the patients' biomedical signal and information can be done entirely using visible light, regardless of the time as dimmed LED is still capable of transmission. Biomedical signals such as ECG and photoplethysmograph (PPG) are different in nature, and the condition of a patient can be determined through 


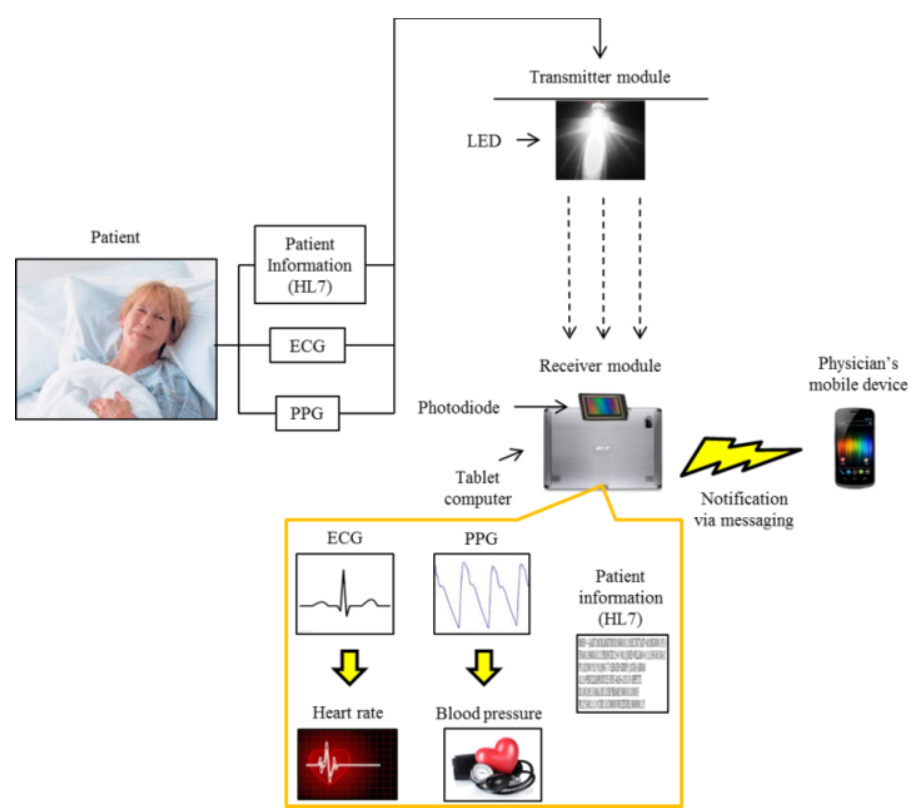

Fig. 1. The proposed system architecture of the mobile health-monitoring system using VLC transmission.

the extraction and analysis of these signals. A communication channel is required to be established to allow the monitoring person to send a notification any physicians when an abnormality is detected. Figure 1 shows the system architecture. PPG and ECG signals, along with the patient information are simultaneous transmitted. The transmission requires both a transmitter module and a receiver module. The acquired information is then displayed and analyzed using a mobile device. Subsequently, heart rate is derived from the ECG signal while arterial blood pressure is estimated through the PPG signal. Finally, a message can be sent to any physician using the function embedded within the application itself.

\subsection{Line-of-sight system configuration}

A line-of-sight system configuration for VLC is implemented in our system. Cui et al. did an experiment to study the relationship between the received optical power and the off-axis angle, $z$ [12]. The off-axis angle is defined as the angle between the transmitter-receiver line and the normal axis of the transmitter. Based on the results from the experiment, a linear decrease in the received optical power is detected as $\mathrm{z}$ is increased. This creates the need to align the receiver at the surface normal to the LED source, in order to receive the maximum optical power at the receiver.

\subsection{Transmitter module}

The transmitter module of the system consists of a modulation circuit and a LED. The LED functions as both an illuminator and transmitter. The LED is used to transmit information by switching between the on-and-off states in a very fast manner. Furthermore, the switching frequency of the LED must be high enough to avoid any flickering that might jeopardize the safety of the human eyes. The modulation scheme implemented in this system is the on-off keying (OOK) non-return-to-zero (NRZ) modulation scheme. OOK NRZ is a part of amplitude-shift keying (ASK) modulation which represents 


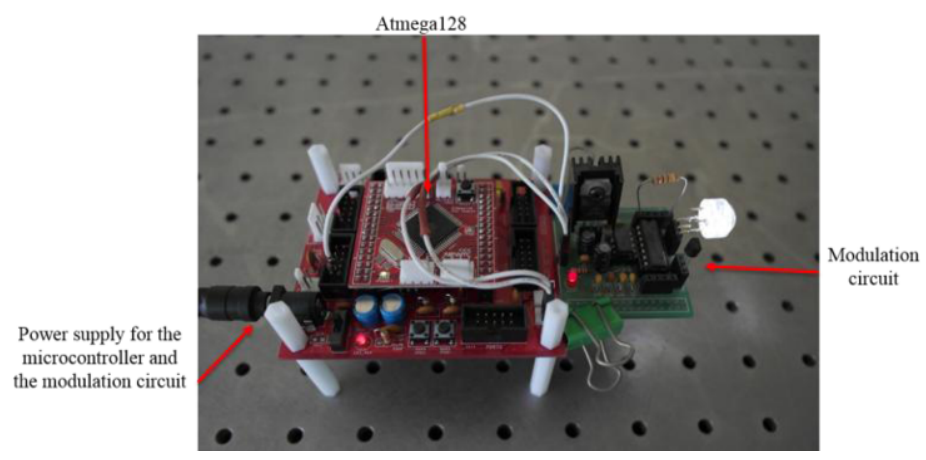

Fig. 2. VLC transmitter module.

the digital information through the presence and absence of the carrier wave. In our design, an LM555 timer is used to generate the carrier wave at a frequency of $200 \mathrm{kHz}$, to ensure that the LED remains illuminated in the view of any observers present. A microcontroller, Atmega128, is used to interface the data to be transmitted with the transmitter module. The modulation process is performed through the use of an NAND gate. Subsequently, a transistor is installed as an optical driver for the LED, to limit the current that flows through the LED. Figure 2 shows the transmitter module used in our proposed system.

\subsection{Receiver module}

A receiver module that is compact in size and portable is designed. The requirements are essential to attach the receiver module on a mobile device. The designed receiver is shown in Figure 3. The receiver module comprises of a demodulation circuit and a microcontroller. Since both the demodulation circuit and the microcontroller require a different amount of voltages to operate, a single battery unit along with two voltage boosters is included. The demodulation circuit consists of various components. A surface-mount silicon PIN photodiode S5107 from Hamamatsu, Japan is chosen as the photodetector for the receiver module. It has an active area of $10 \mathrm{x} 10 \mathrm{~mm}^{2}$, high sensitivity, and wide spectral response ranging from $320 \mathrm{~nm}$ to $1100 \mathrm{~nm}$. The photodiode converts the incident light into an electrical signal. However, the converted signal is often weak and plagued by noises. Hence, there is a need

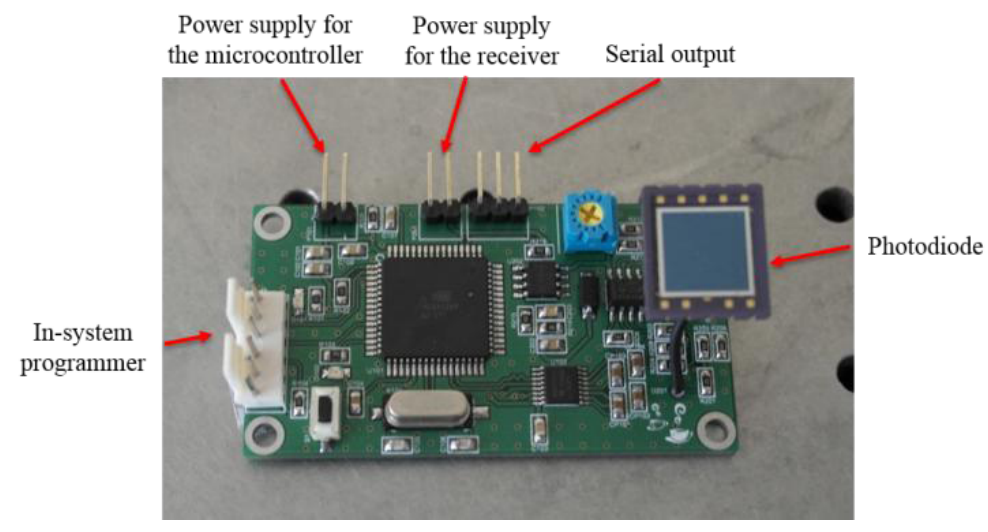

Fig. 3. VLC receiver module. 
for signal conditioning to be performed on the retrieved signal before further processing. First, the electrical signal undergoes amplification. Then, the amplified signal is passed through an envelope detector to demodulate the data signal from the carrier wave. A low-pass filter is implemented subsequently to remove any remaining high-frequency noise. Finally, a voltage comparator is used to transform the signal into digital format, before passing it to the microcontroller for further processing. The microcontroller functions as an intermediate device to serially transmit the data under the RS-232 standard protocol, to another device as a personal computer (PC).

\section{Communication protocol and software design for mobile monitoring}

\subsection{Data package design}

Conventionally, applications that implemented VLC have been associated with the transmission of information using a single LED [13-15]. However, this is largely inconvenient in the healthcare environment. This is due to the need to monitor and evaluate various healthcare information, such as ECG and PPG signals, concurrently to provide a more accurate and efficient prognosis. In our system, we devised a method to simultaneously transmit multiple pieces of healthcare information using only a single white LED. Through the fixation of each data into a single data packet, all the data can be carried over via a single channel. However, there is a limitation to this method as an overloaded data packet might decrease the data transfer rate. The method of transmission is asynchronous for our proposed system. No common clock is applied in the transmission of data. This is especially useful for the transmission of data at an irregular interval. Asynchronous operation relies on the 'start' and 'stop' bits of a data packet to identify the incoming data.

The data packet design in our study is shown in Figure 4. The data packet starts with a synchronization (SYNC) byte, 0xFF. It allows the receiver to know that there is an incoming data. The SYNC byte is then followed by a header byte, 0x80. The purpose of the header byte is to represent the starting point of a data packet. A packet size field is included, to indicate the size of the data included in this data packet. This is crucial as there is a need to dynamically change the size of the data packet, because a large data packet containing the ECG signal, PPG signal and patient information could lead to a higher probability of data loss. This phenomenon can be observed by measuring the packet error rate (PER) using Eq. (1).

$$
\text { PER }=\frac{\text { Number of incorrectly received or erroneous data packets }}{\text { Number of transmitted data packets }}
$$

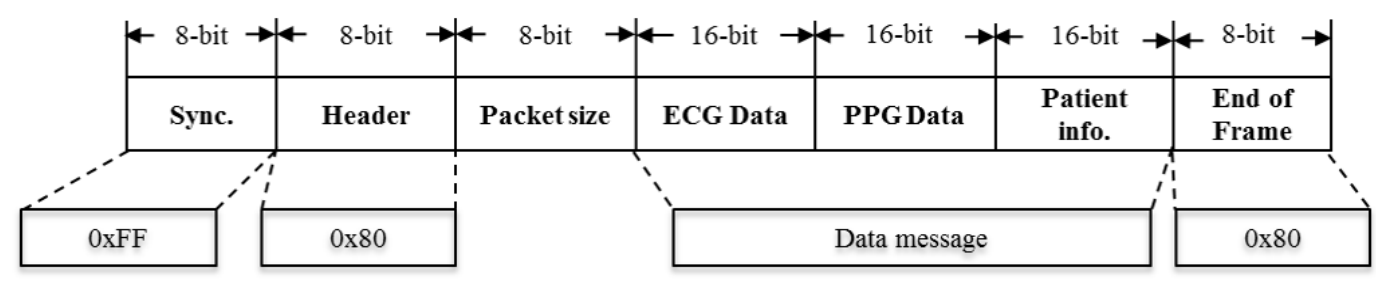

Fig. 4. Data packet design. 
An algorithm is constructed to check for the presence of any text data to be transmitted before the construction of the data packet. The algorithm is designed to constantly check for the presence of any character within the patient information to be transmitted. In the case where the patient information has been fully transmitted, the data packet size can be reduced to contain just the ECG and PPG signals. The dynamic design of the data packet allows the system to only transmit information that is necessary. The payload field contains the data to be transmitted. Finally, the end of a frame is included to signify the end of the data packet.

\subsection{Heart rate}

Heart rate of a person is a vital parameter to be measured in the healthcare industry. The main reason is due to the various conditions that can be identified through the heart rate. ECG signal is often being used to measure heart rate, especially in the healthcare center. The consecutive R-to-R (RR) interval of the ECG signal is an important characteristic that can be used to evaluate the heart rate of a person $[15,16]$. Eq. (2) is used to compute the heart rate. It is measured in beats per minute while the distance is measured in seconds. In our proposed system, the ECG signal is sampled and evaluated in real-time. The information is shown on the application developed, providing the convenience for a physician to monitor and diagnose.

$$
\text { Heart rate }(\text { bpm })=\frac{60}{R R \text { intervals }(s)}
$$

\subsection{Arterial blood pressure}

Arterial blood pressure is often used to determine whether a person is suffering from hypertension. Hypertension is a cardiovascular disease that does not show any immediate symptoms, and if left untreated over a long period, will lead to dire consequences [17]. Arterial blood pressure is measured between each heartbeat, and can vary between a maximum and minimum pressure. Hence, it is categorized into systolic and diastolic blood pressure. Systolic blood pressure (SBP) is measured when the heart contracts and blood are pushed through the arteries. Diastolic blood pressure (DBP) is measured when the heart rests between beats, relieving the arteries off the pressure. PPG signal has long been associated in the determination of arterial blood pressure [17,18]. In our proposed system, the arterial blood pressure is by computed by extracting a certain temporal characteristic from the PPG signal, known as diastolic time (T2) [19]. The derived characteristic can then be applied in Eqs. (3) and (4), to measure SBP and DBP respectively. Each of the linear equations contains two unknown constants, and he or she can be determined independently using the linear regression approach.

$$
\begin{aligned}
& \mathrm{SBP}=a_{S B P} \times \mathrm{T} 2+b_{S B P} \\
& \mathrm{DBP}=a_{D B P} \times \mathrm{T} 2+b_{D B P}
\end{aligned}
$$




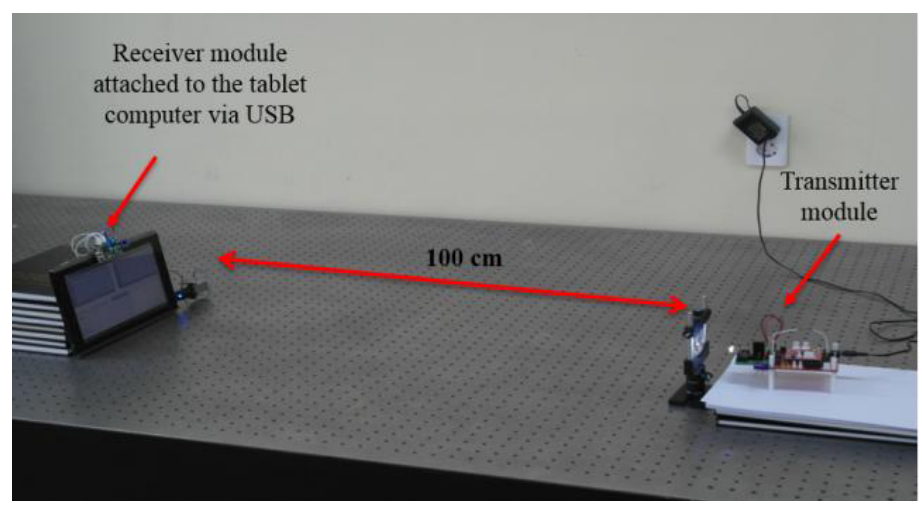

Fig. 5. Experimental setup for the VLC system.

\section{System evaluation}

The reliability and integrity of the proposed system are tested by carrying out numeral experiments to collect results. The VLC system is configured for unidirectional, or one-way, data transmission. The biomedical signals used in the experiment are obtained from PhysioNet [20]. PhysioNet is a database containing a large collection of physiologic signals contributed by researchers and institution around the world, for the purposes of study, research and development. The data transfer rate is set at $2.5 \mathrm{kbps}$, with all the indoor lighting turned-on. Patient information is arranged according to the Health Level Seven (HL7) standard, which is a standard protocol used in the exchange of information between applications in the healthcare industry. A single plano-convex lens is used to increase the luminous intensity of the $1-\mathrm{W}$ single LED used in the experiment, thus extending the transmission distance of the VLC. The experimental setup with LOS configuration and distance of $100 \mathrm{~cm}$ between the transceivers is shown in Figure 5. A tablet computer, Acer Iconia Tab A500 (Acer Inc., Xizhi, New Taipei City, Taiwan), is used in this experiment. The receiver is attached on the tablet computer as shown in Figure 5.

The concept to simultaneously transmit all the information using a single data packet is evaluated by first transmitting both the biomedical signals and patient information. Each time before the size of the data packet is fixed, the presence of any patient information to be transmitted is determined. This is to ensure the payload size of the data packet contains only data that is required. After the patient information has been fully transmitted, the size of data contained within the data packet is reduced from six bytes to four bytes. To justify the need to reduce the size of the data packet, PER for different sizes of payload in the data packet is measured, by transmitting 1000 data packets for each separate cases. Any data not received or wrongly received are counted as packet loss. The results are shown in Table 2 . The receiver will constantly be on standby to lookout for any incoming data packet by identifying the SYNC byte. Then, the microcontroller will transfer the demodulated data to a tablet computer via the serial-to-USB connection. Figures 6(a) and 6(b) shows a screen capture with and without the transmission of patient information, respectively. The measurement of the heart rate and arterial blood pressure are initiated after the transmission of the patient information.

According to Table 2, it can be deduced from the results that a larger data packet contributes to a higher PER. This is especially true when the data packet only contain two bytes of data, where a PER of less than $1 \%$ is detected. The same phenomenon is observed in Figure 6(a), where the biomedical 
signals are distorted when three distinct pieces of healthcare information are received simultaneously. Figure 6(b) shows that after the transmission of the patient information has been halted, much clearer view of the biomedical signals is obtained. Also, the use of the proposed algorithm also increases the sampling rate of the biomedical signals. Sampling rate is an important parameter for the evaluation of the biomedical signals. A higher sampling rate results in an increase in the accuracy of the evaluation of the biomedical signals. In a large data packet, each corresponding data needs to wait for the other data to be included into the data packet before transmission. This generated a certain amount of delays. By reducing the number of data for each transmission, the delay is also reduced.

Table 2

PER measurements based on varying packet size

\begin{tabular}{lll}
\hline Packet size (bytes) & $\begin{array}{l}\text { Number of erroneous data packets detected } \\
\text { over } \mathbf{1 0 0 0} \text { data packets transmitted }\end{array}$ & PER (\%) \\
\hline 2 & 9 & 0.9 \\
\hline 4 & 16 & 1.6 \\
\hline 6 & 20 & 2.0 \\
\hline
\end{tabular}

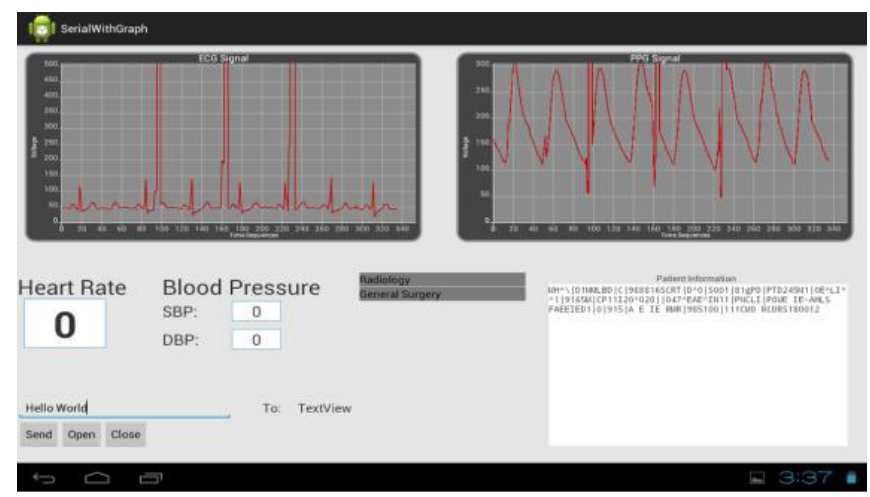

(a)

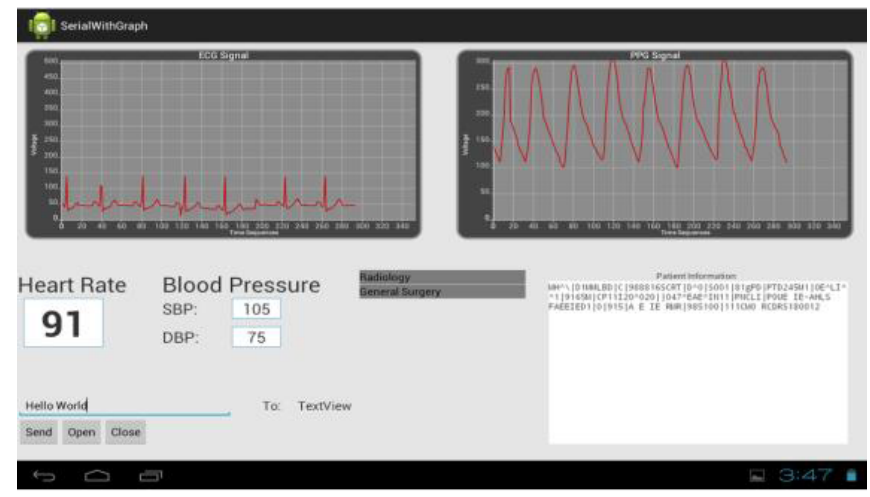

(b)

Fig. 6. Screen capture of the monitoring application where (a) the biomedical signals and patient information are transmitted simultaneously, (b) only the biomedical signals are transmitted. 


\section{Conclusion}

A mobile-health monitoring system using VLC is proposed for the advantages and convenience of the visible light to transmit healthcare information. Biomedical signals and patient information are transmitted simultaneously using a single channel of visible light. By dynamically changing the size of the data packet, the PER of the received data is reduced, thus increasing the reliability of the system. With the help of a mobile device, the proposed system enables innocuous and portable monitoring of a patient's condition. The transmission of the ECG and PPG data can be used for further evaluation, where the heart rate and arterial blood pressure are measured. This is crucial towards the diagnosis of a patient. Furthermore, a notification function is included, by utilizing the capability and ecosystem of the Android operating system. A message can be instantaneously sent to notify any relevant person in case of an emergency during the monitoring process.

\section{Acknowledgement}

This research was supported by Basic Science Research Program through the National Research Foundation of Korea (NRF) funded by the Ministry of Education (2012R1A1B3004140).

\section{References}

[1] New Straits Time, South Korea may band incandescent light bulbs production, imports, http://www.nst.com.my/latest/south-korea-may-ban-incandescent-light-bulbs-production-imports-1.320314, 20th December 2013.

[2] U. S. Department of Energy, Part 1: Review of Life-Cycle Energy Consumption of Incandescent, Compact Fluorescent, and LED Lamps, http://apps1.eere.energy.gov/buildings/publications/pdfs/ss1/2012_LED_Lifecycle_Report.pdf, 20th December 2013.

[3] S.S. Muhlen, D. Davis, B. Segal and G. Vazquez, New challenges in controlling EMI in hospitals, Proceedings of International Federation for Medical \& Biological Engineering, 2008, 834-837.

[4] H. Hong, Y. Ren and C. Wang, Information illuminating system for healthcare institution, Proceedings of International Conference on Bioinformatics and Biomedical Engineering, 2008, 801-804.

[5] K.S. Tan, I. Hinberg and J. Wadhwani, Electromagnetic interference in medical devices: Health Canada's past current perspectives and activities, IEEE International Symposium on Electromagnetic Compatibility, 2001, 1283-1284.

[6] H. Elgala, R. Mesleh and H. Haas, Indoor broadcasting via white LEDs and OFDM, IEEE Transactions on Consumer Electronics 55 (2009), 1127-1134.

[7] H. Haas, High-speed wireless networking using visible light, Optoelectronics \& Communications, 2013.

[8] J. Li, Z. Haiying, H. Kun-Mean and D.V. Christophe, Ubiquitous health monitoring and real time cardiac arrhythmias detection: A case study, Bio-Medical Materials and Engineering 24 (2014), 1027-1033.

[9] S. Challa, G. Geethakumari and C.S.N. Prasad, Patient data viewer: An Android application for healthcare, Annual IEEE India Conference, 2011, 1-4.

[10] S. Gradl, P. Kugler, C. Lohmuller and B. Eskofier, Real-time ECG monitoring and arrhythmia detection using androidbased mobile devices, Annual International Conference of the IEEE Engineering in Medicine and Biology Society, 2012, 2452-2455.

[11] S. Alqassim, M. Ganesh, S. Khoja, M. Zaidi, F. Aloul and A. Sagahyroon, Sleep apnea monitoring using mobile phones, IEEE 14th International Conference on E-health Networking, Application \& Services, 2012, 443-446.

[12] K. Cui, G. Chen, Z. Xu and R.D. Roberts, Line-of-sight visible light communication system design and demonstration, 7th International Symposium on Communications Systems Networks and Digital Signal Processing, 2010, 621-625.

[13] G. Pang, Information technology based on visible light LEDs for optical wireless communications, IEEE Region 10 Conference, 2004, 395-398. 
[14] A. Cailean, B. Cagneau, L. Chassagne, S. Topsu, Y. Alayli and J.-M. Blosseville, Visible light communications: Application to cooperation between vehicles and road infrastructures, IEEE Intelligent Vehicles Symposium (IV), 2012 $1055-1059$.

[15] T. Araki, H. Tatsumi, T. Suzuki and K. Yamada, Application of LED visible light communication signaling for the visually impaired, IEEE International Conference on Systems, Man, and Cybernetics, 2010, 2808-2813.

[16] A. Arcelus, M. Sardar and A. Mihailidis, Design of a capacitive ECG sensor for unobtrusive heart rate measurements, IEEE International International Instrumentation and Measurement Technology Conference, 2013, 407-410.

[17] G. Fortino and V. Giampà, PPG-based methods for non-invasive and continuous blood pressure measurement: An overview and development issues in body sensor networks, IEEE International Workshop on Medical Measurement and Applications, 2010, 10-13.

[18] I.C. Jeong, J.I. Ko, S.O. Hwang and H.R. Yoon, A new method to estimate arterial blood pressure using photoplethysmographic signal, 28th International Conference of the IEEE Engineering in Medicine and Biology Society, 2006, $4667-4670$.

[19] Y.-Y. Tan, S.-J. Jung and W.-Y. Chung, Health monitoring PC mouse with PPG sensor, Proceedings of the 15th International Symposium on Olfaction and Electronic Nose, 2013.

[20] PhysioNet, The research resource for complex physiologic signals, http://www.physionet.org/, 20th December 2013. 